Cell Research (2002); 12(2):157-160

http://www.cell-research.com

\title{
MINIREVIEW
}

\section{Get effective polyclonal antisera in one month}

\author{
Yuan Xin HU, Ju Yuan GUO, Lu SHEN, Yan CHEN, Zu Chuan ZHANG, Yong Lian ZHANG*
}

State Key Laboratory of Molecular Biology, Institute of Biochemistry and Cell Biology, Shanghai Institutes for Biological Sciences, Chinese Academy of Sciences, 320 Yue Yang Road, Shanghai 200031, China

\begin{abstract}
According to the traditional immunization procedure, after the first injection of the sample A (emulsion of aimed antigen and Freund's complete adjuvant) to immunize rabbit, successive injections of the sample B (emulsion of aimed antigen and Freund's incomplete adjuvant) were followed every 2-4 weeks. In general, high titer of the corresponding polyclonal antisera will be observed after 4-5 injections of sample B in 3-4 months. This report presents a simply modified procedure that was able to stimulate the antisera formation in one month and achieve enough avidity to satisfy either Western blot or immunohistochemistry analysis. It just applied an additional injection of the sample A to the rabbit at the 3rd day after the primary immunization injection. You could gain the high titer of the antisera right after the first sample B injection in one month. This method has produced the desired results in three different recombinant antigens with different molecular weight (5.9 KD-55 KD) expressed from prokaryotic or eukaryotic cells.
\end{abstract}

Key words: Polyclonal antisera preparation, Modified immunization method.

\section{INTRODUCTION}

Polyclonal antisera are a useful tool in characterization of a gene product, especially for the new gene discovery at the protein level. Although it has been a great deal of commercial kits available for getting the aimed recombinant protein and for their purification as well. However, immunization is still regarded as a limit step in the whole process because of its time-consuming and sometime the antisera will not be effective in the immunostaining by some unknown reasons.

According to the traditional immunization method, after the first injection of the emulsion of aimed antigen and Freund' s complete adjuvant as primary immunization, three or four injections of the emulsion of aimed antigen and Freund' $\mathrm{s}$ in-

* Corresponding author, E-mail: yonglz@sunm.shcnc.ac.cn Received April-5-2002 Revised April-10-2002 Accepted April12-2002 complete adjuvant will be followed two or four weeks each as subsequent immunization. The whole process usually takes three or more months to achieve high titer antisera individually[1],[2]. We happened to find that just addition of an extra injection with the same sample (emulsion of aimed antigen and Freund抯 complete adjuvant) at the 3rd day after the primary immunization, and then followed by one subsequent immunization injection in the first month will be adequate to achieve as high titer of polyclonal antisera as that of the antisera obtained in three months or more by the traditional method. We observed that the titer of all the antisera raised by our modified method in the three cases was about 7.660.5 times higher than that raised by the traditional method in one month. The positive immunoreaction of the three polyclonal antisera against their respective antigen was observed by Western blot analysis. Moreover, the immunostaining ability of the antisera against either GCNF/RTR or Bin2a showed posi- 
tive results.

\section{MATERIALS AND METHODS}

\section{Animals}

Animals (New Zealand white rabbit, BALB/c mouse and SD rat) were purchased from The Animal Center of the Chinese Academy of Sciences. Healthy male New Zealand rabbits aged 3-6 months (body weight $\sim 2.5 \mathrm{~kg}$ ) lived in animal house at least for 3-4 days for adapting to the new circumstance.

\section{Polyclonal antisera production}

Sample A: Emulsify your aimed protein $200-300 \mu \mathrm{g}$ in PBS with same volume of the Freund' $\mathrm{s}$ complete adjuvant $(3-4 \mathrm{mg} / \mathrm{ml}$ heat-killed Bacillus tubercle in lanolin : mineral oil=2:5 [v/v] ) in $1 \mathrm{ml}$ total volume.

Sample B: Same as injection solution I but no Bacillus tubercle.

The modified immunization method: At the first day, $1.5 \mathrm{ml}$ blood was collected by bleeding rabbits from the marginal vein of the ear for preparing the pre-immunized-sera as negative control. Afterwards, $1 \mathrm{ml}$ of sample A in 30-50 $\mu \mathrm{l}$ per each site was injected intradermally on the back and proximal limbs of the rabbit[1, 3 , 4]. On the 3rd day, repeat the above injection once to strengthen the first immunization stimulation. On the 28th day, $1 \mathrm{ml}$ of sample B was injected by the same way. On the 35 th day, $0.5 \mathrm{ml}$ blood was collected by the same way used before for checking its titer of the antisera by ELISA. Harvest the antisera from the arteriae carotis.

Traditional immunization method: Exactly same as the modified method, except no additional sample A injection on the 3rd day. Besides, it needed several sample B injections in every 2-4 weeks successively.

\section{Enzyme-linked immunosorbent assay (ELISA)}

The titers of the polyantisera were determined by indirect ELISA[5],[6] with respective pre-immunized serum as negative control, the antisera as deals. Read the plate by sights and using a spectrophotometer at $450 \mathrm{~nm}$ to determine the titer. Repeat three times.

\section{Immunostaining of antisera against proteins of the GCNF/RTR and Bin2a}

The immunohistochemistry of paraffin-embedden sections from Bouins-fixed adult mouse testis and para- formaldehydefixed $45 \mathrm{~d}$ rat epididymis was done by using $\mathrm{ABC}$ kit (from SinoAmerican Biotechnology Company). The dilution of primary antisera was 1:1000 and the latter step was according to the manufacture' s direction. Antisera staining was developed by 3 , 3'-diaminobenzidine (in GCNF/RTR case according to Ref.[7]). Slides were counterstained by Eosin complex in GCNF/RTR case and Hematoxylin in Bin2a (from MERCK company).

\section{RESULTS AND DISCUSSION}

The modified immunization method is efficient and reliable to different protein antigens with different molecular weight

During the process of raising the antisera of GCNF/RTR, we happened to find that, with two continual injections of the emulsion of antigen and Freund' s complete adjuvant (sample A) within three days, it resulted in a much higher titer of the antisera in about one month. Fig $1(\mathrm{GCNF} / \mathrm{RTR})$

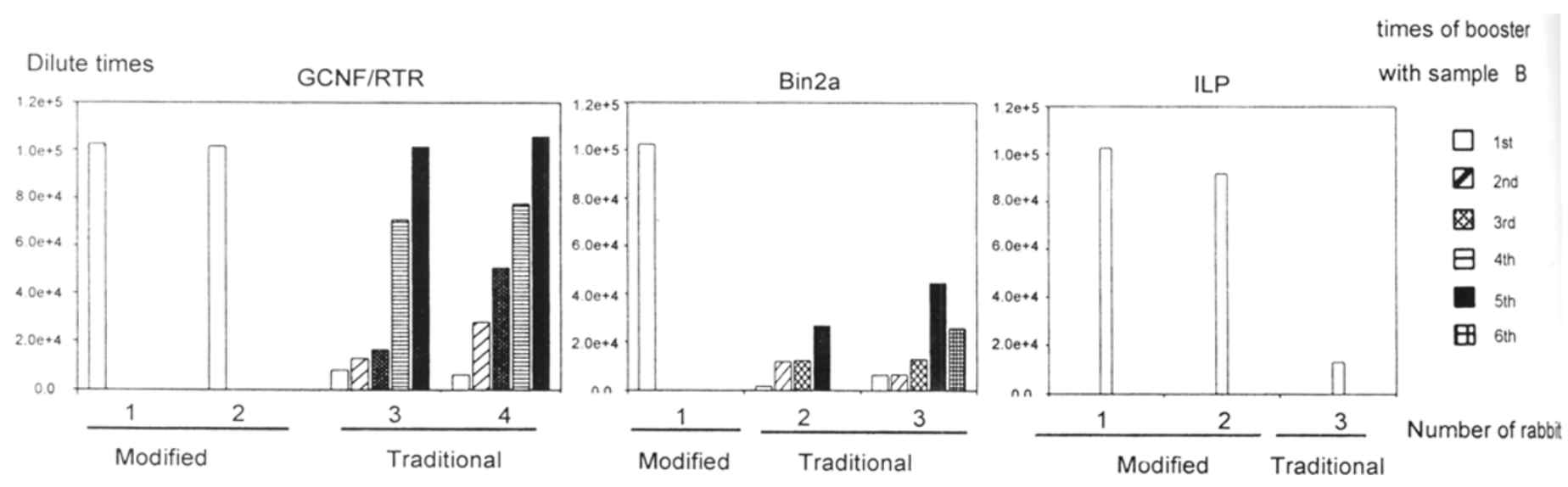

Fig 1. Comparison of the titer of the antisera raised by the modified and traditional immunization method The abscissa represents the number of the rabbit. The ordinate represents the titer of the antisera measured by ELISA (the dilute times adopt to the Scientific Count Method, e.g. $1.2 \mathrm{e}+5$ presents $1.2 \times 10^{5}$ ). The different columns represent the number of the immunized times by sample B (emulsion of antigen and Freund' $\mathrm{s}$ incomplete adjuvant). 


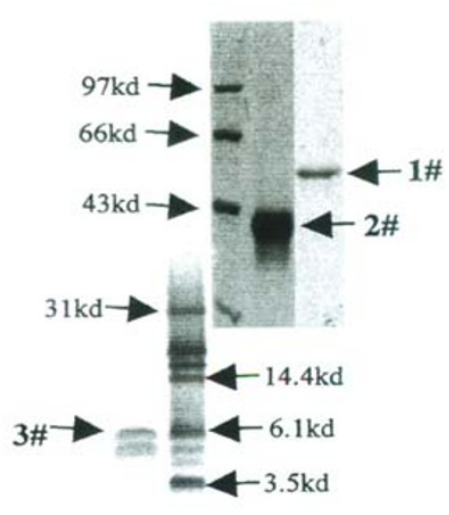

Fig 2. The SDS-PAGE pattern of the three purified proteins expressed from E.coli or yeast $1^{\#}$. GCNF/RTR, wt. 55,000 Daltons; $2^{\#}$. Bin2a, wt. 34,000 Daltons; $3^{\#}$. ILP, wt. 5900 Daltons. showed that it needed another 4 months more time to get the same titer of the antisera derived from the two rabbits immunized by traditional method after additional 6 times of booster with sample B. For making sure if it is just a fortuitous phenomenon or it is of common significance. We used several proteins as antigen with different molecular weight and expressed from different cells. As shown in Fig 2, the molecular weight of the three different antigens spanned about ten folds i.e. the 55KD GCNF/RTR (germ cell nuclear factor/retinoid receptor-related testis-associated receptor)[8],[9] and a $38 \mathrm{KD}$ newly discovered gene product Bin2a fragment expressed in E. Coli and the $5.9 \mathrm{KD}$ ILP(insulin like peptide) [10] fragment expressed in yeast. The modified immunization method was paralleled with the tradi-

\section{Testis (GCNF/RTR)}
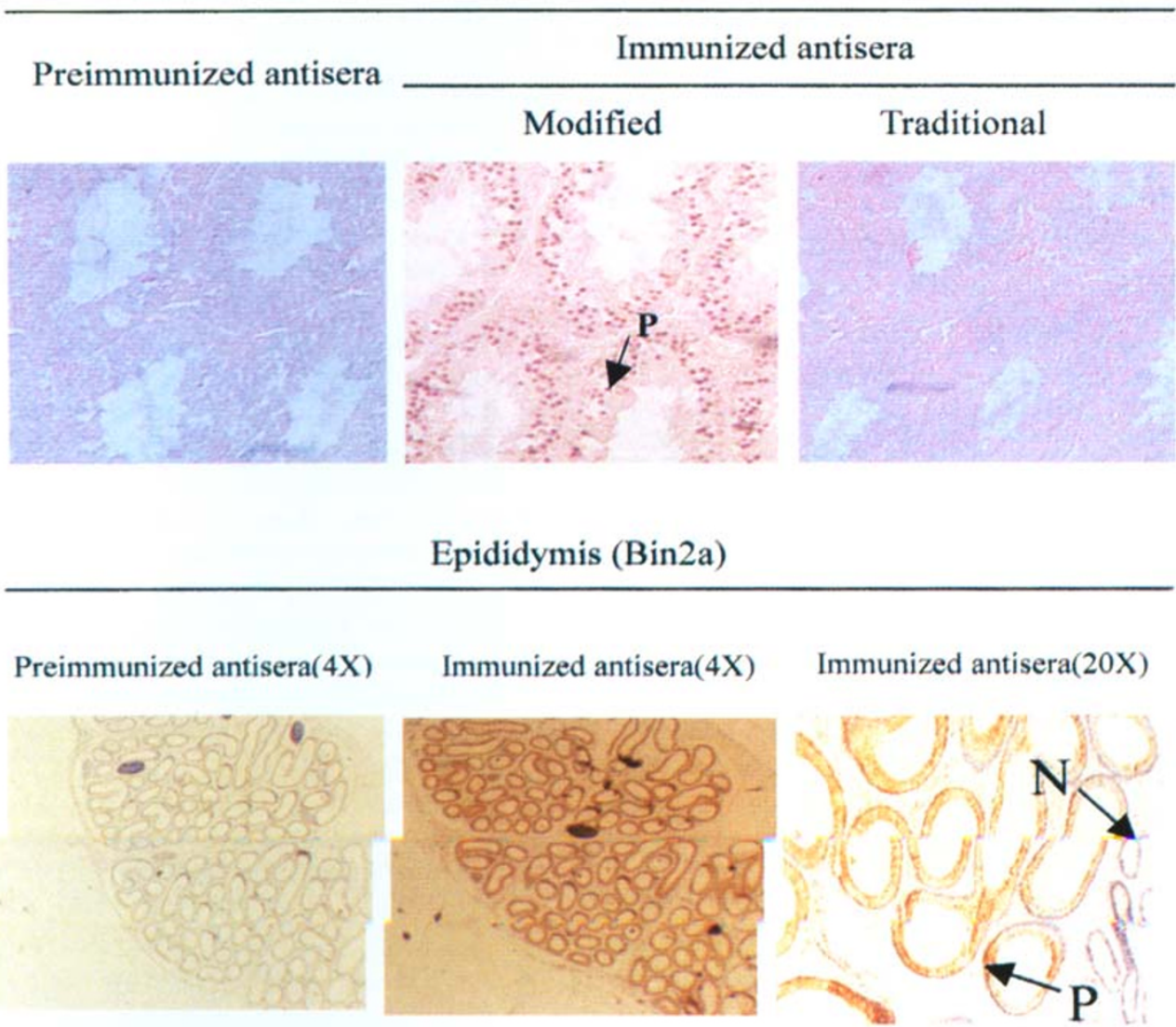

Fig 3. Immunostaining of antisera against GCNF/RTR and Bin2a

In GCNF/RTR case, the immunostaining was restricted in the nuclei of the primary, secondary spermatocytes and round spermatids in the middle zone of mouse testis. The Bin2a stained in the cytoplasma of the epithelial cells of the rat epididymis. $\mathrm{N}$ : negative immunostaining; P: positive immunostaining. 
tional one. We did observe that the titers of all the antisera raised by our modified immunization method in the three cases was obviously higher than those raised by the traditional method in one month. The superiority was at least 7.6 times higher in the ILP case and even 60.5 times higher in the Bin2a case. Besides, in the Bin2a case, even 4 times more of sample B injections boosted to the two rabbits (number 2 and 3 ) in another two month time, the titer increased too slow to be continued. The reliability of the modified method was confirmed again by another three case (two insulin receptor fragment with M.W. around 14,000 Dalton and a fragment of Carboxylesterase M.W.34,000 Dalton) in our institute recently (data not shown).

The antisera raised by the modified immunization method were effective used in western blot and immunohistochemistry assays

Quality tests were followed to evaluate those polyclonal antisera rasied by our modified immunization methods. It makes sense that all the antisera against their respective antigen derived from either the traditional method or the modified one showed positive immuno-reaction in the Western blot analysis (data not shown). However, the divergence appeared in their immunostaining ability evaluation. It could be seen in Fig 3 that only the antisera raised by the modified method (no matter it against GCNF/ RTR or Bin2a) gave positive response but not the traditional method one.

All these results indicated that with no extra additional materials or special procedure needs, just a minor modification of the immunization method, was not only be able to stimulate the antisera formation but also achieve enough avidity to satisfy immunohistochemistry ananlysis by unknown reason. In another words, in one month or even less, you can get a high titer antisera for using in both Western blot and immunostaining analysis.

\section{ACKNOWLEDGEMENTS}

We would like to thank Peifang Ping, Baozhen Peng and Dr. Xinxiu Yang for their helps in immunization and ELISA. This work was funded by the National Natural Sciences Foundation of China, No. 39893320, the "973" Basic Research Funding Scheme of China (G 1999055901) and the Shanghai Institute for Biological Sciences, Health Science Center Research Funding.

\section{REFERENCES}

[1] Frederick M. ausubel, Roger Brent, Robert E. Kingston, et al. Production of Polyclonal Antisera in Rabbits. In: Short Protocols in Molecular Biology, 4th, Wiley Press, Toronto, 1999; 11:22-5.

[2] Jonathan A Green, Margaret M Manson. Production of Polyclonal Antisera, In: Methods in Molecular Biology. Humana Press, Totowa, New Jersey 1998; 80:1-4.

[3] J Udith L Vaitukaitis, production of Antisera With Small Dose of Immunogen. In: Methods in Enzymology, Academic Press, New York, 1981; 73:46-52.

[4] Paul H Maurer, Hugh J Callahan. Protein and Polypeptides as Antigen, In: Method in Enzymology, Academic Press, New York, 1980; 70:49-63.

[5] JR Crowther. Indirect ELISA. In: ELISA, Theroy and Pratice, Methods in Molecular Biology, Humana Press Totowa, New Jersey, 1995; 42:131-60.

[6] JR Crowther. The ELISA Guidebook. In: Methods in Molecular Biology, Humana Press Totowa, New Jersey 2000; 149:9-44.

[7] Joe C Adams. Heavy Metal Intensification of DAB-based HRP Reaction Product, The Journal of Histochemistry and cytochemistry, 1981; 29:6, p. 775.

[8] Fang Chen, Austin J Cooney, Yaolin Wang, Simon W Law, Bert W O扢alley. Cloning of a novel Orphan Receptor (GCNF) Expression during germ cell development, Molecular Endocrinology, 1994; 1434-44.

[9] Takahisa Hirose, Deborah A. O' Mrien and Anton M. Jetten, RTR: a new member of the nuclear receptor superfamily that is highly expressed in murine testis,gene, 1995; 152:247-51.

[10] Shu Jin Chan, Qiuping Cao, Donald F. Steiner, Evolution of the insulin superfaimly: Cloning of a hybrid insulin/insulin-like growth factor cDNA from amphioxus. Proc Natl Acad Sci USA 1990; 87:9319-23. 